\title{
Primeval Jets from Young Stars
}

\author{
T. P. Ray \\ Dublin Institute for Advanced Studies, 31 Fitzwilliam Place, Dublin 2, Ireland \\ email: tr@cp.dias.ie
}

\begin{abstract}
The jet phenomenon lasts at least a million years for young, solar-like, stars and it occurs during a wide variety of young stellar object (YSO) phases. This includes the period when the source is highly embedded (Class 0) to when it becomes optically visible for the first time as a classical T Tauri star (Class II). Here I briefly discuss some of the properties of jets from the youngest objects.
\end{abstract}

Keywords. Protostar, YSO, jet, accretion, outflow, Herbig-Haro object, interstellar medium

\section{Introduction}

Low mass stars like the Sun are thought to form from so-called pre-stellar cores, cold $(\approx 10 \mathrm{~K})$ substructures within molecular clouds that have no internal heating source but are instead warmed by the interstellar radiation field (Kirk et al. 2007). Most of the mass of the young star is then built up during the highly embedded Class 0 phase when powerful outflows accompany high accretion rates. This is illustrated, for example, by the mm source at the centre of the dramatic Herbig-Haro (HH) 211 outflow (Lee et al. 2009). Despite the extent and power of this outflow, the core mass of the YSO is less than that of a brown dwarf. As accretion declines, the protostar enters the Class I phase characterised by rising flux at infrared wavelengths while the source remains optically invisible. Such obscuration is caused by the presence of a circumstellar disk in combination with a dusty envelope. In the Class II phase the photosphere of the newborn star is directly visible for the first time: it is now a classical T Tauri star or a Herbig Ae/Be star if it has a higher mass. The Class 0 , I and II phases are thought to last about $10^{5}, 5.10^{5}$ and $2.10^{6}$ years respectively (Evans et al. 2009).

Distinguishing between these various evolutionary phases can however be difficult. Modelling the observed spectral energy distribution (SED) relies on a combination of photospheric (stellar), disk and envelope emission in which multiple scattering and reprocessing of photons is important. Moreover the envelope may be partially cleared out, i.e. contain a cavity as a result of the outflow, and the disk may be flared (Robitaille et al. 2006). Add to this a random inclination angle of the disk/outflow axis to our line of sight and immediately it is seen that what might be regarded as a Class I source if viewed from a particular angle may look like a Class II source when seen from another. An added complication in constructing an SED is non-steady accretion and thus variable luminosity (Evans et al. 2009).

Some of these ambiguities may be removed by using the properties of associated outflows. For example, through a combination of proper motion studies and spectroscopy, the actual 3-D direction of a jet may be established (e.g. Caratti o Garatti et al. 2009). Assuming this is along the disk axis, the inclination angle of the disk to the line of sight may be found. Jets are also fossil records of how active their source has been in the past: with proper modelling, they can at least give us clues on the variation in activity level over thousands of years (i.e. over dynamical timescales). In particular, if outflow rates 
(as measured through the jet) are a proxy for accretion and accretion rates decline with time, then jets may help us distinguish between the various evolutionary phases.

In what way do the youngest (primeval) jets from Class 0 sources differ from those of more evolved protostars? In the past observations of such outflows were largely confined to studying low energy molecular transitions, e.g., the CO J=1-0 line. Such transitions trace slowly moving ambient gas, i.e. gas propelled forward by the much more highly collimated underlying atomic jet. Instead if we wish to observe the latter, at velocities close to the gravitational escape velocity from the star $\left(\approx 100 \mathrm{kms}^{-1}\right)$, we have to study the outflow in the mid-infrared using for example the Infrared Spectrograph (IRS) on Spitzer. The reason for this is that the standard permitted and forbidden atomic lines in the optical and near-infrared (e.g., [SII] $\lambda \lambda 6716,6731$ and $[\mathrm{FeII}] 1.64 \mu \mathrm{m}$ ) are too heavily obscured by ambient dust. Spitzer studies of outflows from Class 0 sources not only show that an underlying atomic component is present but that the mass loss rate in such jets is much higher than those from less embedded sources of equivalent mass (e.g., Dionatos et al. 2009).

Recently it has been found that many jets from young stars contain significant amounts of dust (Podio et al. 2009)). The presence of dust is not measured directly but is instead inferred from line ratios of refractory to non-refractory species. While depletion values are not as high as in the ISM, the gas in a jet has not attained solar abundances, at least close to the source. The dust itself appears to be intrinsic rather than entrained from the jet's surroundings, as there is very little dynamical evidence for entrainment. Moreover the dust seems to be only gradually destroyed via the mild internal jet shocks, as expected from grain models (Jones et al. 1994). We have only started to observe abundance ratios in jets from Class 0 sources. Again the problem is that the jet itself is relatively embedded and so the normal optical or even near-infrared lines cannot be used. We thus have to revert to mid-infrared wavelengths and initial observations also suggest large amounts of dust in such flows (Dionatos et al. 2009).

In the future we can look forward to studying the youngest and most embedded jets using telescopes like ALMA and the Mid-Infrared Instrument (MIRI) on the James Webb Space Telescope (JWST). In particular the MIRI IFU, given its good spatial and spectral resolution, will be particularly useful for studying the dynamics, chemical evolution, and basic properties of atomic jets from Class 0 sources.

\section{Acknowledgements}

TPR would like to thank the organisers for an excellent Joint Discussion and to acknowledge support from Science Foundation Ireland (Grant 07/RFP/PHYF790).

\section{References}

Caratti o Garatti, A., Eislöffel, J., Froebrich, D., Nisini, B., Giannini, T., \& Calzoletti, L. 2009, $A \mathscr{G} A, 502,579$

Dionatos, O., Nisini, B., Garcia Lopez, R., Giannini, T., Davis, C. J., Smith, M. D., Ray, T. P., \& DeLuca, M. 2009, ApJ, 692, 1

Evans, N. J., et al. 2009, ApJS, 181, 321

Jones, A. P., Tielens, A. G. G. M., Hollenbach, D. J., \& McKee, C. F. 1994, ApJ, 433, 797

Kirk, J. M., Ward-Thompson, D., \& André, P. 2007, MNRAS, 375, 843

Lee, C.-F., Hirano, N., Palau, A., Ho, P. T. P., Bourke, T. L., Zhang, Q., \& Shang, H. 2009, ApJ, 699, 1584

Podio, L., Medves, S., Bacciotti, F., Eislöffel, J., \& Ray, T. P. 2009, arXiv:0907.3842

Robitaille, T. P., Whitney, B. A., Indebetouw, R., Wood, K., \& Denzmore, P. 2006, ApJS, 167, 256 